\title{
Parametric Spectrum Analysis of Backscattered Ultrasound Signals for the Characterization of Particles in Suspensions
}

\author{
Michael Vogt, Michael Deilmann \\ KROHNE Messtechnik GmbH, Ludwig-Krohne-Str. 5, 47058 Duisburg, Germany
}

\begin{abstract}
Summary
Suspensions, i.e. heterogeneous mixtures of solid particles in liquids, play an important role in various industries and applications. Especially in-line methods for analyzing the concentration and the size of the solid particles in a given suspensions are of large interest in chemistry and process industry, for example for monitoring and control of sedimentation processes in wastewater treatment plants. Further examples include the analysis of drilling mud, paints, and others. Ultrasound-based methods are a powerful tool for this task, because the ultrasound waves are scattered at the solid particles inside the liquid phase and, different from optical techniques, also allow for measurements with opaque media. In the case of a particles size much smaller than the ultrasound wavelength, as is given in many practical applications, Rayleigh backscattering occurs. Accordingly, the intensity of the scattered ultrasound waves is proportional to the fourth power of the frequency, i.e. the scattering phenomenon is largely frequency-dependent. This can advantageously be utilized for characterizing suspensions by a spectral analysis of the backscattered waves (echo measurement) or of the transmitted waves (transmission measurement). The goal of the work presented here was to develop a robust method for characterizing the concentration and the size of the particles by employing a parametric approach. This is achieved by fitting an analytical model to the spectra of acquired echo signals and by deriving quantitative parameters thereof. The proposed concept has been tested and validated by means of experimental echo measurements with ultrasound in the $2 \mathrm{MHz}$ range.
\end{abstract}

Keywords: Ultrasound analysis, Rayleigh scattering, parameter extraction, quantitative measurement, model fit.

\section{Introduction}

Ultrasound-based methods can very advantageously and quite easily be applied for the characterization of fluids (liquids, gases). Especially in the case of homogeneous media, various acoustic parameters can be quantitatively measured by means of transmission and reflection measurements with ultrasound [1]. Using the measured speed of sound, acoustic impedance, and attenuation coefficient, it is possible in many applications to estimate further parameters like the mass density or the concentration of a mixture of known liquids [2].

In also interesting other applications, however, inhomogeneous media are given. This includes various aerosols (liquid particles in a gas, 'wet gas'), and suspensions (solid particles in a liquid). The problem in this case is that the propagating ultrasound waves are largely scattered (or reflected) inside the medium. For this reason, an ultrasound wave shows large and random amplitude variations after it has been propagated through the scattering medium. On the other hand, echo measurements can be made in this case and allow for analyzing the scattering behavior of the medium. Many works have already been published in this field [3-8]. A fundamental challenge with regard to the characterization of inhomogeneous scattering media remains to derive quantitative parameters from the ultrasound measurement signals in a robust manner.

In this contribution, a parametric model approach is used to describe the spectral characteristics of backscattered ultrasound waves. The model is fitted to the magnitude spectra of acquired echo signals, and quantitative parameters are derived from the fitted model. Experiments have been performed in order to evaluate the potential of this concept for a further analysis of different suspensions. Results fg these measurements will be presented and discussed in detail. 


\section{Ultrasound Backscattering Measurement}

In Fig. 1, a setup for ultrasound backscattering measurements of suspensions is illustrated.

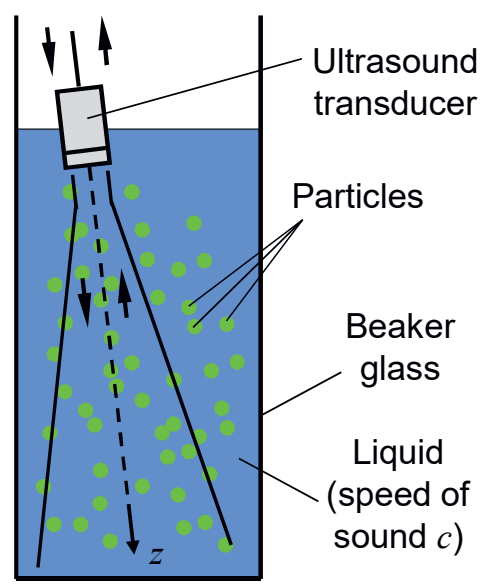

Fig. 1: Ultrasound backscattering measurement setup (illustration).

A single-element ultrasound transducer is directly immersed into the suspension, which is a heterogeneous mixture of solid particles in a liquid. In this monostatic configuration, the transducer is connected to a pulser / receiver electronic and used for both, the transmission and the reception of ultrasound. Accordingly, ultrasound waves, which are backscattered at the particles inside the suspension, are acquired over time of flight (TOF) by means of pulse-echo measurements.

The positions of the particles in the liquid are random, and the echo signal amplitude over TOF is a random variable for this reason. To allow for a statistical analysis under these conditions, the echo measurement is preferably repeated several times with different spatial arrangements of the particles. For the below described experimental measurements, this has been achieved by continuously stirring the suspension (using a magnetic stirrer).

Each given TOF $t$ of the echo signals $x(t)$ can be linked to an axial distance $z=c / 2 \cdot t$ by taking the speed of sound (SOS) $c$ of the suspension into account, see Fig. 1. With a relatively small concentration of the particles, the SOS is approximately equal to that of the liquid (for example water: $c=1480 \mathrm{~m} / \mathrm{s}$ ).

The goal behind the below presented signal processing strategies is to realize a robust spectral analysis of the digitized echo signals. Special care is taken in this context to only include the echo signals, which are caused by the backscattering at the particles, in the analysis and to exclude other disturbing echoes. In practice, the latter can especially be given by multiple reflections inside the transducer ('transducer ringing'). Furthermore it might happen quite easily that also significant echoes from further objects appear. An example could be reflections from the floor or walls of the beaker glass in Fig. 1, which contains the suspension. Each acquired echo signal is time-gated over TOF prior to the further signal processing in order to analyze only the backscattering from inside the suspension.

A general drawback of using ultrasound is that the ultrasound waves are also strongly scattered at gas bubbles, which might be given in the liquid. Accordingly, the applicability of ultrasound-based analysis systems is generally limited to suspensions with no relevant content of gas bubbles.

\section{Ultrasound Scattering Properties}

The focus of this work is on analyzing small particles with a mean particles diameter $d$ that is much smaller compared to the wavelength $\lambda$ of the utilized ultrasound waves. With the SOS $c$ of the liquid, this condition can be described as follows for a given ultrasound frequency $f$ :

$$
d \ll \lambda \text {, with: } \lambda=c / f
$$

As an example, a wavelength $\lambda \approx 740 \mu \mathrm{m}$ is given in water for $f=2 \mathrm{MHz}$. The condition in (1) is fulfilled for many practically relevant suspensions, and Rayleigh scattering occurs at the particles in this case. As a consequence, the incident ultrasound wave is diffusely scattered into all directions [9].

The acoustic backscattering coefficient of the particles depends largely on the mismatch between the acoustic impedances (Al) of the particles and of the liquid. Furthermore, this parameter also depends on the mean particles diameter $d$, and it largely increases with frequency in the case of Rayleigh scattering. Accordingly, the amplitude (and so the intensity) of the echo signal as well as its spectral characteristic depend on the particles size and concentration. If echo signals from different suspensions but with the same type of particles, i.e. consisting of the same material, are analyzed, the aforementioned parameters allow for analyzing both, the size and the concentration of the particles.

The scattering at the particles is a first reason for the attenuation of the ultrasound waves, which propagate through the suspension. Generally, a second reason is viscous losses inside the liquid. In most cases, however, and especially with a large particles concentration and a large acoustic mismatch of the particles, the attenuation given by the scattering is much larger as compared to that given by the viscosity of the liquid. 
Specifically for Rayleigh scattering, the intensity of the scattered ultrasound waves is proportional to the fourth power of the frequency. Under the assumption of a uniform distribution of the random particles positions, the echo signal amplitude is a random variable, which is described by the Rayleigh distribution [9].

\section{Spectral Analysis of Backscattered Waves}

In this section, echo signal processing concepts for a robust and reliable spectral analysis of the backscattered ultrasound waves from the suspension are presented and discussed.

In a first step, the amplitude spectrum of each digitized and time-gated echo signal is calculated by using the Fast Fourier Transform (FFT). Thereafter, the amplitude spectra obtained from multiple repeated measurements with changing spatial arrangement of the particles are averaged to account for the statistical variations of the echo signal amplitude. Next, a parametric model is fitted to the mean amplitude spectrum. In a final step, quantitative parameters are derived from the fitted model to characterize the backscattering properties of the suspension. In Fig. 2, a Gaussian model for describing the amplitude spectrum $|X(\omega)|$ over the (angular) frequency $\omega$ of an ultrasound echo signal $x(t)$ is shown:

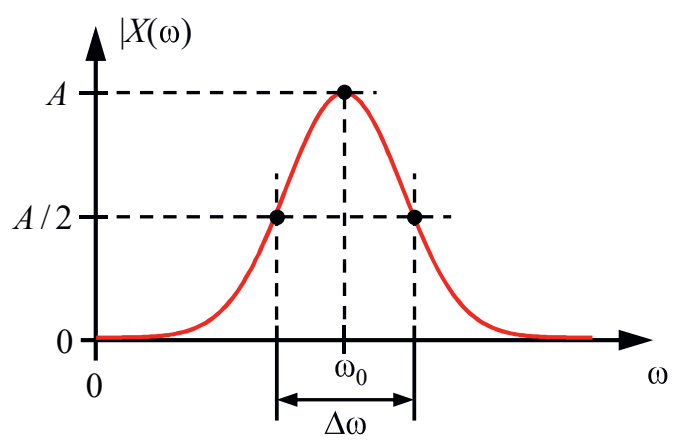

Fig. 2: Gaussian spectrum model; definition of quantitative parameters derived thereof: Amplitude $A$, center frequency $\omega_{0}$, bandwidth $(-6 \mathrm{~dB}) \Delta \omega$.

As will be shown below, the Gaussian model usually fits quite well to amplitude spectra of 'real' ultrasound echo signals obtained in practical experiments. The Gaussian model in Fig. 2 can be analytically described as follows:

$$
|X(\omega)|=A \cdot \mathrm{e}^{-k \cdot\left(\omega-\omega_{0}\right)}, \quad \Delta \omega=2 \cdot \sqrt{\ln (2) / k}
$$

The parameter $A$ in (2) denotes the amplitude, $\omega_{0}$ the (angular) center frequency, and $\Delta \omega$ the (angular) bandwidth $(-6 \mathrm{~dB})$ of the echo signal.
(The constant $k$ in (2) is only used for an easier writing of the equations.) Accordingly, the amplitude spectra of measured echo signals are completely described by these three parameters after the Gaussian parametric model has been fitted.

\section{Fitting of Parametric Spectrum Model}

The concept of the further processing steps is to fit the analytic spectrum model to the mean amplitude spectrum obtained from the echo measurements. The parameters of the fitted model (amplitude, center frequency, and bandwidth) are then used for analyzing the suspension, as they are expected to be closely linked to the particles concentration and size, which are the interesting physical parameters of the suspension.

A further important advantage of using the Gaussian model in (2) is that an analytical and unambiguous mathematical solution exists to calculate the least square fit to any given spectral measurement data [10]. This solution can be derived quite easily, and the computational effort for calculating the fit is very low.

As has already been discussed, the extracted model parameters are used below to characterize the suspension and, accordingly, to differentiate between different suspensions. Reference measurements on suspensions with known properties can be utilized in this context in order to calibrate the system.

\section{Realized Measurement Setup}

We have realized the laboratory measurement setup shown in Fig. 3.

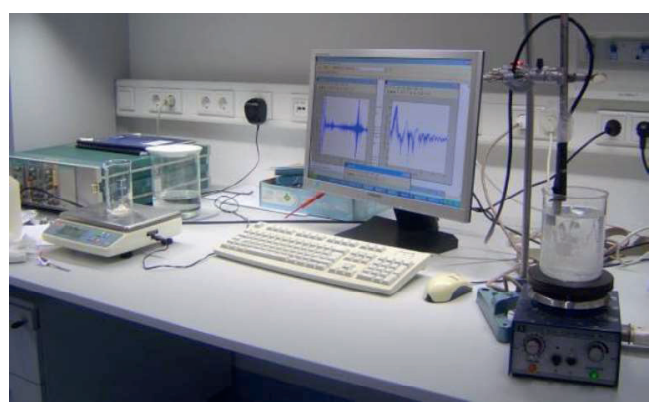

Fig. 3: Realized measurement setup (laboratory equipment).

A single-element ultrasound piston transducer with a nominal center frequency $f_{0}=2 \mathrm{MHz}$ and $D=10 \mathrm{~mm}$ aperture diameter has been used for all the below presented measurements. A pulser / receiver is used in this setup for performing pulse-echo measurements. The echo signals are directly digitized with an analog-to-digital converter (ADC) board with 14 bit amplitude resolution and $10 \mathrm{MHz}$ 
sampling frequency (Spectrum Systementwicklung Microelectronik $\mathrm{GmbH}$, Grosshansdorf, Germany). The digitized echo data is transferred into a personal computer (PC) for the further digital processing and analysis. The utilized transducer has a relatively large bandwidth $(-6 \mathrm{~dB})$ of $700 \mathrm{kHz}$, what allows for a good time-gating and for analyzing the echo signals over a relatively wide frequency range.

\section{Measurement Samples}

The above introduced concepts for processing the echo signals have been evaluated with results of various practical experiments. Measurements were performed using two different samples of suspensions, both containing the same type of plastic particles (i.e. consisting of the same material), but with different particles size:

- Sample \#1: Finely granulated particles

- Sample \#2: Coarsely granulated particles

Five different suspensions were prepared by using tap water and different particles concentrations (mass-volume fractions of 6,12 , 18,24 , and $30 \mathrm{~g} / \mathrm{l}$ ). A magnetic stirrer has been used to achieve a largely homogeneous spatial distribution of the particles in the suspensions. In Fig. 4, two exemplarily measurement situations with the two different samples \#1 and \#2, respectively, are shown with the same particles concentration of $12 \mathrm{~g} / \mathrm{l}$. a)

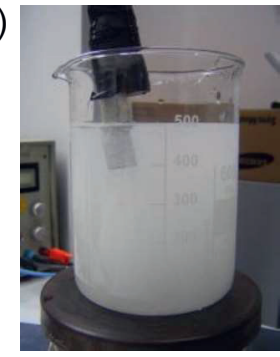

b)

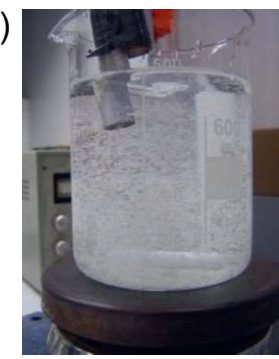

Fig. 4: Suspensions with particles of different granularity and size; particles concentration of $12 \mathrm{~g} / \mathrm{l}$ : a) Finely granulated particles (sample \#1), b) coarsely granulated particles (sample \#2).

\section{Examples of Recorded Echo Signals}

In Fig. 5, one exemplarily recorded echo signal is shown (blue curves) for each of these two cases. The echo signals have been plotted over the axial distance $z$, which has been calculated by using a SOS $c=1480 \mathrm{~m} / \mathrm{s}$ for the water. At small distances (up to about $15 \mathrm{~mm}$ ), echoes with a very large amplitude can be identified, which are caused by multiple reflections inside the transducer. Here, the
ADC has actually been saturated on purpose with the transducer ringing in order to keep the quantization noise small and to achieve a large dynamic range for the interesting backscattering echoes. The latter can clearly be identified at larger distances and have much smaller amplitudes compared to the transducer ringing.
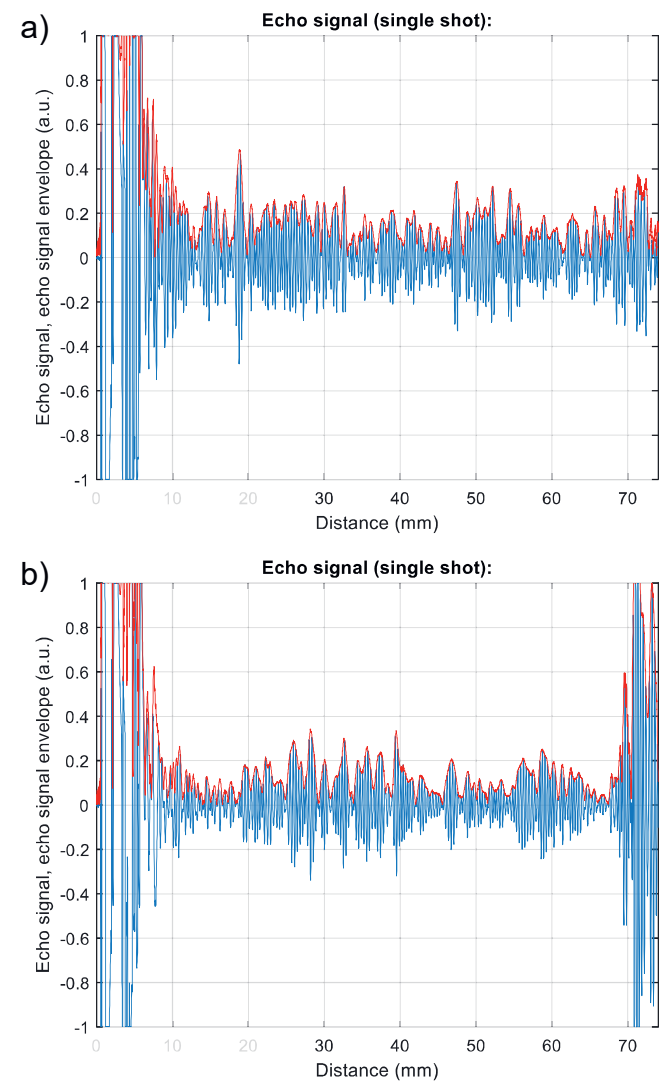

Fig. 5: Examples of acquired echo signals (blue curves) and echo signal envelopes (red curves); particles concentration of $12 \mathrm{~g} / \mathrm{l}: \quad$ a) Finely granulated particles (sample \#1), b) coarsely granulated particles (sample \#2).

At axial distances of about $70 \mathrm{~mm}$ and larger, echoes from the floor of the beaker glass, see Fig. 4, can be seen. The red curves in Fig. 5 show the echo signal envelopes, which have been calculated in each case as the magnitude of the analytical echo signal, obtained by using the Hilbert transform of the digitized echo signal. The comparison of Figs. 5 a) and b) shows that the mean echo signal amplitude is larger in the case of sample \#1 (finely granulated particles) as compared to the sample \#2 (coarsely granulated particles).

A series of 512 consecutive echo measurements has been performed with each of the investigated samples with different particles concentration. In Fig. 6, the envelopes of the 
consecutively acquired echo signals are shown. The utilized gray-scale coding shows the logarithmic echo signal amplitude over a dynamic range of $50 \mathrm{~dB}$. Each column in the images in Fig. 6 represents one single echo signal envelope over the axial distance $z$.

a)

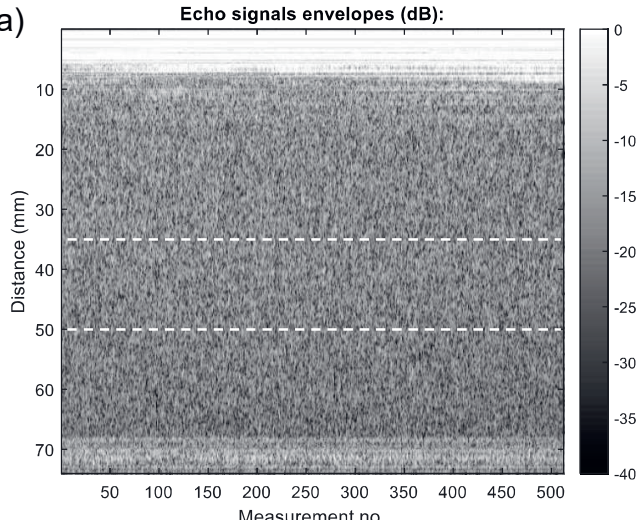

b)

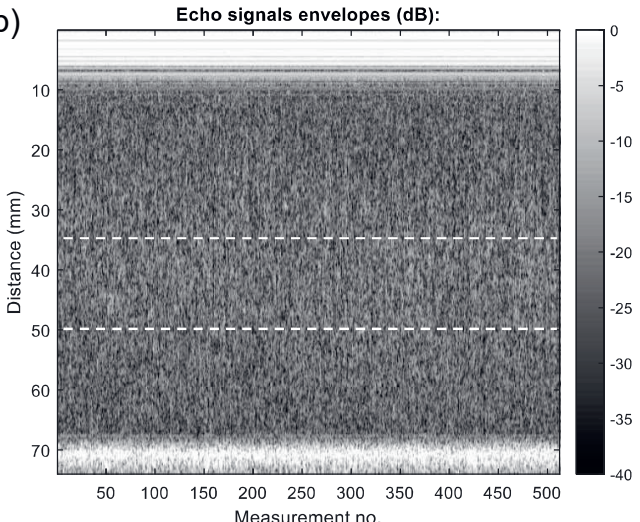

Fig. 6: Examples of acquired echo signals envelopes for consecutively repeated measurements; particles concentration of $12 \mathrm{~g} / \mathrm{l}$ : a) Finely granulated particles (sample \#1), b) coarsely granulated particles (sample \#2).

The transducer ringing can now be seen much better, appearing as constant echoes over the consecutive measurements (i.e. appearing as horizontal lines). The spatial arrangement of the scattering particles changes from one measurement to the next, because of the stirring. Accordingly, the consecutive echo signals are uncorrelated with each other. The random scatterer arrangement can be seen by the very large fluctuations of the echo signals envelopes, which are quite commonly referred to as 'speckle noise' [9].

\section{Series of Measurements}

As described above, measurements have been performed with the two samples \#1 and \#2 and with varying particles concentration. As in the example above, echoes from 512 consecutive measurements have been digitized for each concentration. In the following, the acquired echo signals and the echo signal spectra, as well as results of the further spectral analysis will be presented and discussed.

\section{Echo Signals Envelopes}

The echo signal envelopes of the individual echoes have been calculated separately and averaged over the 512 measurements. The only purpose of averaging the echo signal envelopes was to show the according results in Fig. 7 and to allow for a discussion of these results.
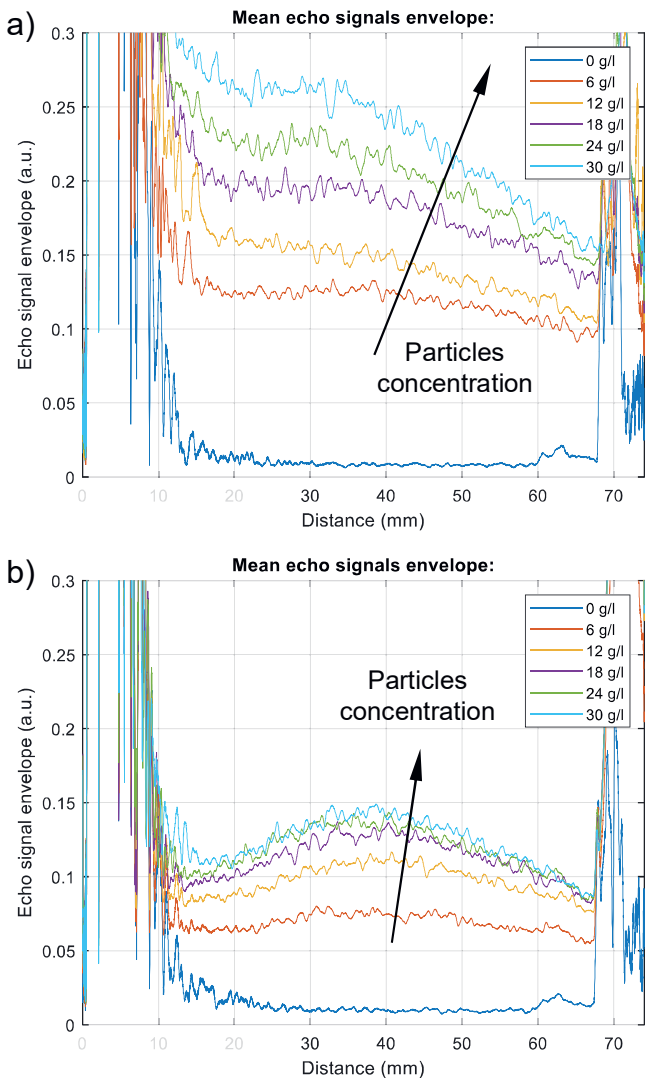

Fig. 7: $\quad$ Mean envelopes of acquired echo signals for varying particles concentration: a) Finely granulated particles (sample \#1), b) coarsely granulated particles (sample \#2).

The comparison of Fig. 7 with Fig. 5 shows that the echo signal fluctuations are very efficiently suppressed by the performed averaging of the uncorrelated 'observations' of the scattering phenomenon. It can be seen in Fig. 7 that the envelope signal increases with increasing particles concentration, what is in agreement with the expectations. For the finely granulated particles (sample \#1), the echo signal amplitude is much larger as compared to the coarsely granulated particles (sample \#2) in the case of comparing the same particles concentrations. 


\section{Echo Signals Spectra}

Each digitized echo signal has been timegated prior to the spectral analysis in order to include only the backscattering from the suspension. A Hanning window function has been applied over the axial distance range from $35 \mathrm{~mm}$ to $50 \mathrm{~mm}$ for this purpose, see the dashed lines in Fig. 6.

In a first step, the amplitude spectrum of each individual time-gated echo signal has been calculated using the FFT. Thereafter, the resulting 512 echo signal amplitude spectra have been averaged. This has been done here again in order to suppress the random echo signal fluctuations prior to fitting the analytical Gaussian spectrum model in each case. The resulting mean amplitude spectra are shown in Fig. 8 (marked by the stars in the figures) for a frequency interval from $1 \mathrm{MHz}$ to $3 \mathrm{MHz}$.
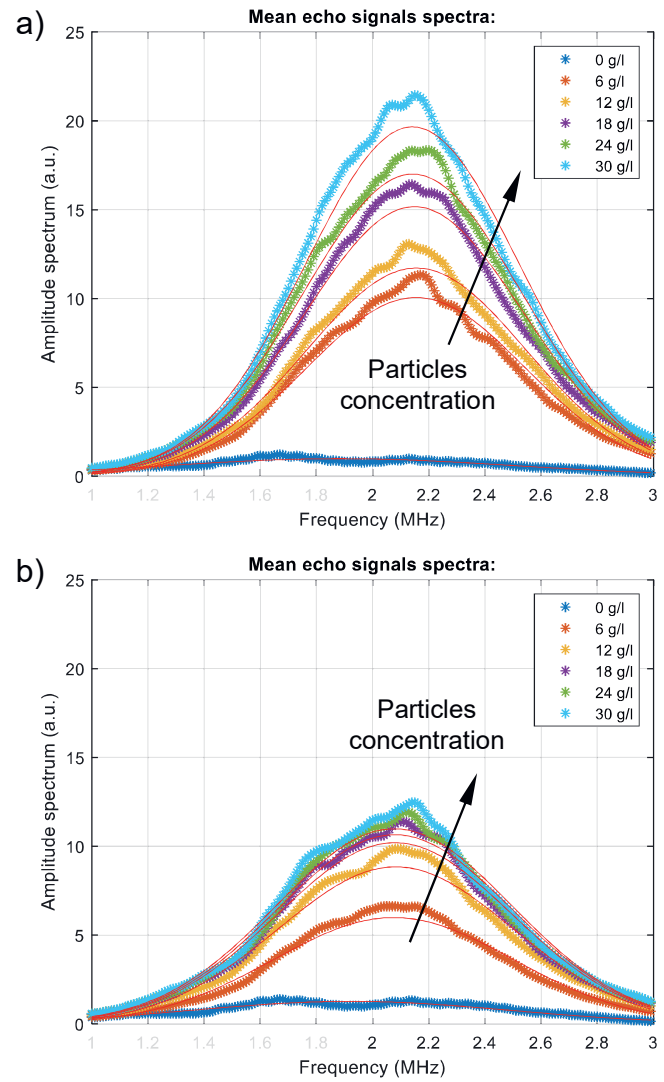

Fig. 8: $\quad$ Mean amplitude spectra of acquired echo signals for varying particles concentration: a) Finely granulated particles (sample \#1), b) coarsely granulated particles (sample \#2).

The spectra are predominantly given by the passband of the utilized ultrasound transducer, and this is in good agreement with the nominal center frequency $f_{0}=2 \mathrm{MHz}$ of the transducer. The plotted spectra show a $(-6 \mathrm{~dB})$ bandwidth $\Delta f \approx 0.7 \mathrm{MHz}$ of the transducer.
In another step, the parametric Gaussian spectrum model according to (2) has been fitted to each of the individual mean amplitude spectra. Only the measured amplitude spectra in the frequency interval from $1 \mathrm{MHz}$ to $3 \mathrm{MHz}$ has been used for the least square fit in order to exclude spectral components with a low signal-to-noise ratio (SNR), see Fig. 8.

The fitted spectra have also been plotted in Fig. 8 (solid lines). It can be seen that the Gaussian model fits actually quite well to the amplitude spectra of the experimentally acquired ultrasound echo signals. The amplitudes $A$ of the fitted spectra, see Fig. 2, show a similar result like the mean echo signal envelopes of the backscattered ultrasound signals in Fig. 7.

\section{Quantitative Spectral Parameters}

The three parameters describing the Gaussian spectrum model (amplitude, center frequency, and bandwidth) have been extracted from the fitted spectra in Fig. 8 in each case. Based on these quantitative data, the potential of these parameters for a differentiation between the two samples \#1 and \#2 of suspensions at varying particles concentration has been evaluated and tested.

In Fig. 9, the two estimated parameters, center frequency and bandwidth, are shown over the given particles concentration for the two samples:

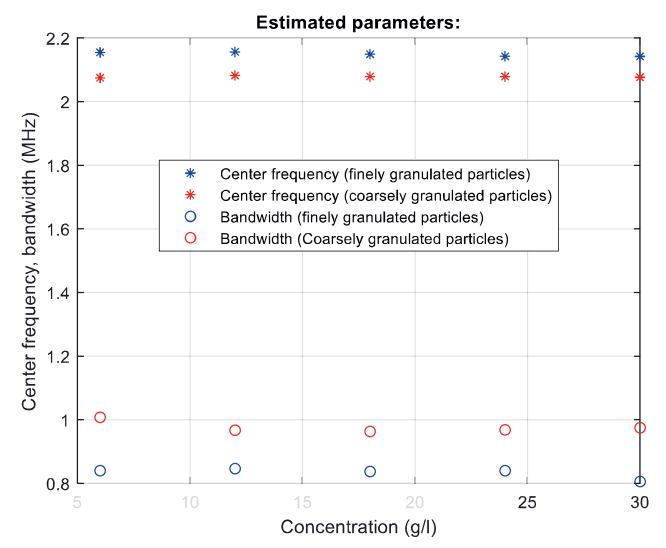

Fig. 9: Estimated center frequency and bandwidth for varying particles concentration: Finely granulated particles (sample \#1), coarsely granulated particles (sample \#2).

Both parameters are largely constant over the concentration, but they are significantly different for the two samples. This is in a good agreement with the expectation that the spectral backscattering properties change with the mean particles size.

In Fig. 10, the same two parameters (center frequency and bandwidth) are now plotted 
against each other. Consequently, this plot represents a two-dimensional parameter-space [2]. Different symbols (stars and circles) have been used to indicate to which of the two samples each data point at varying concentration belongs.

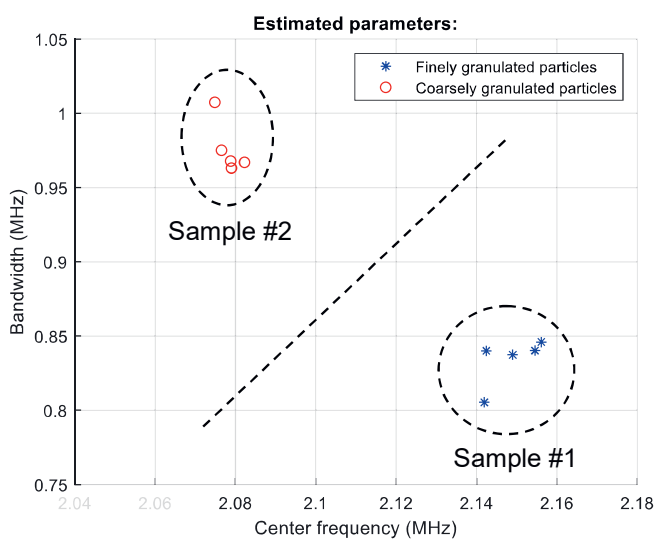

Fig. 10: Two-dimensional parameter space (bandwidth over center frequency): Clustering of data points for the two samples \#1 and \#2, respectively.

It can be seen much better now that the two parameters (center frequency and bandwidth) are largely different for the two samples, and that they are largely independent of the particles concentration. The mismatch in the acoustic impedances (of the particles and the liquid) and the particles concentration, on the other hand, are expected to have a large influence only on the echo signal amplitude.

The data points in Fig. 10 for the varying concentration are building separate and nonoverlapping clusters for the two different samples. Accordingly, a differentiation between these two samples can be made, i.e. the two parameters allow for a classification of the actually given type of sample.

Finally, the intensity $I=A^{2}$ of the backscattered ultrasound waves, has been calculated from the estimated amplitude $A$ (derived from the Gaussian spectrum model) in each single case, see Fig. 11. The plots show that the particles concentration can be derived from the measured intensity by using these results as reference data in terms of a calibration of the system. In order to estimate the concentration of a given unknown sample, the intensity has to be measured and the functions in Fig. 11 have to be inverted. However, the problem is that the actually given type of suspension (finely or coarsely granulated particles) has to be identified prior to the inverse calculation in order to know which of the two reference curves has to be used. But the according classification of the given suspension can be done as discussed above with Fig. 10.

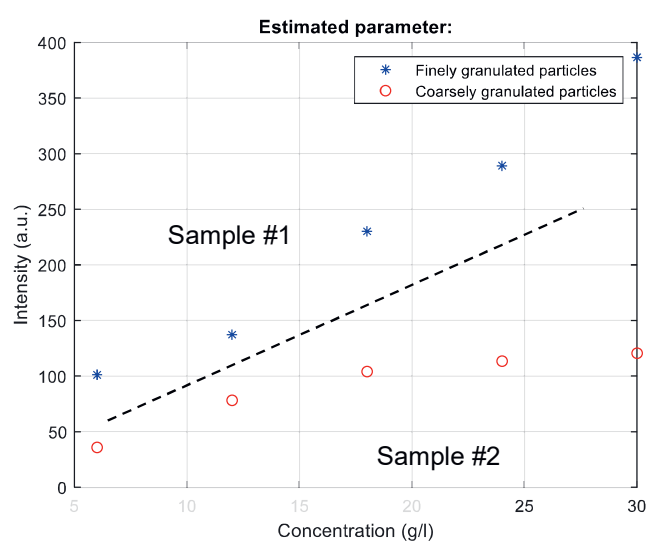

Fig. 11: Estimated intensity of backscattered ultrasound waves for varying particles concentration: Finely granulated particles (sample \#1), coarsely granulated particles (sample \#2).

Consequently, in a more general approach, a given unknown suspension can be characterized with regard to the particles size based on the estimated center frequency and bandwidth, while the particles concentration can be estimated based on the measured echo signal intensity. In any case, however, reference data obtained from calibration measurements with well-defined and known samples would have to be used ('supervised learning') to eliminate the system-specific influences onto the ultrasound echo measurement. Among these effects, the actually given transfer characteristics of the utilized ultrasound transducer is the most important one, which has to be accordingly considered.

\section{Summary and Conclusions}

In this contribution, concepts for analyzing suspensions by means of ultrasound echo measurements have been presented. General aspects of ultrasound echo measurement setups and the realization of a system for pulse-echo measurements with a singleelement ultrasound transducer have been discussed. The fitting of a parametric Gaussian spectrum model to the measured mean echo signal amplitude spectrum has been proposed as a robust approach. Experiments have been performed with two different kinds of suspensions, consisting of the same type of particles, but with different particles size. It has been shown that the quantitative parameters amplitude, center frequency, and bandwidth, which are derived from the fitted model, have a large potential for assessing the particles size as well as the particles concentration of a given suspension. The proposed and 
investigated method might potentially be used for inline analysis of suspensions in various industrial applications.

\section{References}

[1] M. Vogt, H. Ermert: Quantitative Analysis of Liquids and Emulsions by means of HighFrequency Ultrasound (15-35 MHz). IEEE Int. Ultrasonics Symp., 68-71 (2011); doi: 10.1109/ULTSYM.2011.0018

[2] M. Vogt, S. Langener, J. Kunze, T. Musch, $\mathrm{H}$. Ermert: Measurement and Multivariate Analysis of Ultrasound Parameters for Quantitative Characterization of Liquids. IEEE Int. Ultrasonics Symp., 1137-1140 (2012); doi: 10.1109/ULTSYM.2012.0283

[3] S. Wöckel, U. Hempel, R. Weser, B. Wessely, J. Auge: Ultraschallstreuverfahren zur Charakterisierung von Dispersionen mit hohem Partikelanteil. 16. GMA/ITG-Fachtagung Sensoren und Messsysteme, 344-351 (2012); doi: 10.5162/sensoren2012/3.3.2

[4] R. Weser, B. Wessely, S. Wöckel, U. Hempel: Partikelmessung an hochkonzentrierten Dispersionen nach dem UltraschallReflexionsprinzip. 16. GMA/ITG-Fachtagung Sensoren und Messsysteme, 344-351 (2012); doi: 10.5162/10dss2011/6.2

[5] S. Wöckel, U. Steinmann, R. Weser,

B. Wessely: Inline-Prozessüberwachung von Suspensionen mit hohem Feststoffanteil. 11. Dresdner Sensor Symposium, 463-466 (2013); doi: 10.5162/11dss2013/L17

[6] S. Wöckel, R. Weser, U. Hempel, B. Wessely, J. Auge: Monitoring of Highly Concentrated Suspensions by Ultrasonic Scattering. IEEE Int. Ultrasonics Symp., 1133-1136 (2012); doi: 10.1109/ULTSYM.2012.0282

[7] D.J. McClements: Ultrasonic Measurements in Particle Size Analysis. In: Encyclopedia of Analytical Chemistry, (Sept. 2006); doi: 10.1002/9780470027318.a1518

[8] J. Carlson, A. Greenberg: Ultrasonic Measurements of Particle Concentration in a Multiphase Flow. IEEE Int. Ultrasonics Symp., 757-760 (1999); doi: 10.1109/ULTSYM.1999.849510

[9] R.F. Wagner, S.W. Smith, J.M. Sandrik, H. Lopez: Statistics of Speckle in Ultrasound BScans. IEEE Trans. Sonics Ultrasn., vol. 30, no. 3, 155-163 (May 1983) ; doi: 10.1109/TSU.1983.31404

[10] M. Vogt, H. Ermert: In Vivo Ultrasound Biomicroscopy of Skin: Spectral System Characteristics and Inverse Filtering Optimization. IEEE Trans. Ultrason., Ferroelect., Freq. Contr., vol. 54, no. 8, 15511559 (August 2007) ; doi: 10.1109/TUFFC.2007.425 